\title{
Psoriasis as risk factor for non-ischemic dilated cardiomyopathy: a population-based cross-sectional study
}

\author{
Abbas Alshami ${ }^{1}$, Nasam Alfraji ${ }^{1}$, Steven Douedi ${ }^{*}$, Swapnil Patel ${ }^{1}$, Mohammad Hossain ${ }^{1}$, Deborah Alpert ${ }^{2}$ and \\ Dawn Calderon ${ }^{3}$
}

\begin{abstract}
Background: Psoriasis is a chronic inflammatory skin condition commonly associated with psoriatic arthritis, malignancy, diabetes, inflammatory bowel disease, and cardiovascular disease. Several reports and studies have reported an association between psoriasis and non-ischemic dilated cardiomyopathy (NIDCM). We aim to study the relationship between psoriasis and non-ischemic dilated cardiomyopathy in a large population-based study.

Methods: We utilized the Healthcare Cost and Utilization Project National Inpatient Sample 2017 database, which represents a 20\% sample of all payer hospitalizations in the United States. We investigated hospitalizations for patients aged 18 years old or older with diagnoses of any type of psoriasis and non-ischemic dilated cardiomyopathy. Psoriasis, cardiomyopathy, and other comorbidities were identified through their international classification of diseases, 10th revision codes recorded in the discharge record for each hospitalization.

Results: Of a total of $6,084,184$ all-cause admissions, $0.5 \%$ were admissions for patients with psoriasis $(n=32,807)$. Of the patients with and without psoriasis who had non-ischemic dilated cardiomyopathy, after adjusting for age, sex, race, diabetes mellitus, hypertension, alcohol abuse, cocaine abuse, arrhythmias, and obesity in a multivariate analysis, the presence of psoriasis was not significantly associated with non-ischemic dilated cardiomyopathy.

Conclusion: Psoriasis is a chronic autoimmune disorder which carries a higher cardiovascular events and more prevalent traditional atherosclerotic risk factors in comparison to the general population. However, association with non-ischemic cardiomyopathy or NIDCM in particular has not been studied sufficiently. Our study, being one of the first larger studies to assess this correlation, indicated no relationship between psoriasis and non-ischemic dilated cardiomyopathy.
\end{abstract}

Keywords: Psoriasis, Psoriatic arthritis, Non-ischemic dilated cardiomyopathy, Cardiovascular disease, Autoimmune disease

*Correspondence: Steven.Douedi@hmhn.org

${ }^{1}$ Department of Medicine, Jersey Shore University Medical Center, 1945 Route 33, Neptune, NJ 07753, USA

Full list of author information is available at the end of the article

\section{Introduction}

Psoriasis is a chronic inflammatory skin condition which commonly presents with scaly erythematous plaques. It has a worldwide prevalence ranging from 0.5 to $11 \%$ [1]. Psoriasis is an immune mediated disease, with T-Cells, dendritic cells, and cytokines playing a crucial role in skin damage [2]. The most common complication or association with psoriasis is psoriatic arthritis, which involves 
the musculoskeletal system [3]. Other conditions include but are not limited to malignancy, diabetes, inflammatory bowel disease, and several studies have implied a possible relationship between psoriasis and cardiovascular disease [4-13]. While the relationship between psoriasis and cardiomyopathies, especially non-ischemic dilated cardiomyopathy (NIDCM), has been suggested through case reports and small observational studies, the association has not been evaluated using a large sample. This population-based study aims to investigate the correlation between patients with psoriasis and non-ischemic dilated cardiomyopathy.

\section{Methods}

\section{Study design/settings}

We conducted a cross sectional study for adults hospitalized for any cause at any hospital in 2017 in the United States. We utilized the Healthcare Cost and Utilization Project National Inpatient Sample (HCUP-NIS) 2017 database, which represents a $20 \%$ sample of all payer hospitalizations in the United States. These hospitalizations were selected systematically by the Agency for Healthcare Resources and Quality (AHRQ), thus represent all the hospitalizations in the United States in 2017. Notably, these encounters represent hospitalizations, not patients, so multiple potential hospitalizations for the same patient are considered as multiple encounters.

\section{Participants}

We included all hospitalizations for patients $\geq 18$ years old. We divided them to two main groups, admissions with and without psoriasis. Then, we identified admissions with and without NIDCM, as well as other clinical entities, in each main group. Encounters for patients aged $<18$ years old were excluded due to differences in risk factors and pathophysiology of cardiomyopathy between the pediatric and adult populations.

\section{Variables}

The data collected in the database included demographic variables, admission diagnoses, procedures, type of insurance, geographical location, length of stay, and inpatient mortality, among others. Every encounter can include up to 40 diagnoses and are coded using the international classification of diseases, 10th revision (ICD-10 codes).

\section{Outcomes}

We aimed to investigate if psoriasis represents an independent risk factor for NIDCM.

\section{Data measurement}

Admission diagnoses, including psoriasis, NIDCM, and other comorbidities and substances were identified through their ICD-10 codes recorded in the discharge record for each hospitalization. "Appendix" contains a list of the ICD-10s used in this study.

\section{Ethical considerations}

Institutional review board (IRB) is not required to for studies utilizing the national inpatient sample, as it falls under the "limited data sets" category exempted by the HIPAA privacy regulations (http://privacyruleandresear ch.nih.gov). The study was conducted in agreement with the principles of the Declaration of Helsinki.

\section{Statistical methods}

Continuous and categorical variables were described as mean with standard deviation (SD) and frequencies, as appropriate. Chi square and t-test were used to compare categorical and continuous variables, as appropriate. Multivariate analyses were performed to determine if psoriasis represents an independent risk factor for cardiomyopathies. All analyses were done using IBM SPSS Statistics $^{\mathrm{TM}}$ version 25.0 (IBM Corporation, Artmonk, $\mathrm{NY}$ ). An alpha value ( $p$ ) of 0.05 was used to ascertain statistical significance.

\section{Results}

Of a total of $6,084,184$ all-cause admissions, $0.5 \%$ were admissions for patients with psoriasis $(\mathrm{n}=32,807)$.

\section{Baseline characteristics}

Baseline characteristics differed between admissions with and without psoriasis and are summarized in Table 1. Mean age was 61 years with SD of 15.4 years for patients with psoriasis, as opposed to mean age of 57 years with SD of 20.2 years in admission for patients without psoriasis. Around half of the encounters were for female patients, and White was the most prevalent race for both populations. Generally, comorbidities were more prevalent in patients with than without psoriasis.

\section{Psoriasis and NIDCM}

Of all-cause admission records, $0.7 \%$ had NIDCM, as opposed to $0.9 \%$ in patients with psoriasis $(p=0.03)$. However, after adjusting for all the variables listed in Table 1 in a multivariate analysis, psoriasis failed to survive as an independent predictor of NIDCM (Table 2).

When NIDCM was further subdivided into different causes, only viral and alcoholic cardiomyopathies were statistically associated with psoriasis in bivariate analyses (Chi-square test) (Table 3). Multivariable analyses using multivariable logistic regression models showed that psoriasis survived as an independent risk factor for viral CM with marginal statistical significance (adjusted OR 2.3, 95\% CI 1.020-5.131, $p=0.045$ ), while it failed to predict 
Table 1 Baseline characteristics of all admitted patients and patients with psoriasis

\begin{tabular}{|c|c|c|c|}
\hline & $\begin{array}{l}\text { All admissions } \\
\text { Mean (SD) or N (\%) }\end{array}$ & $\begin{array}{l}\text { Admissions with psoriasis } \\
\text { Mean (SD) or N (\%) }\end{array}$ & $p$ value \\
\hline Age (years) & $57.9(20.3)$ & $61.2(15.4)$ & $<0.001$ \\
\hline Sex & & & $<0.001$ \\
\hline Male & $2,554,599(42.2)$ & $16,388(50)$ & \\
\hline Female & $3,496,439(57.8)$ & $16,416(50)$ & \\
\hline Race & & & $<0.001$ \\
\hline White & $3,924,190(67.2)$ & $26,032(79.3)$ & \\
\hline Black & $891,986(15.3)$ & $1782(5.4)$ & \\
\hline Hispanic & $649,121(11.1)$ & $2275(6.9)$ & \\
\hline Asian or Pacific Islander & $161,026(2.8)$ & $668(2)$ & \\
\hline Native American & $361,77(0.6)$ & $195(0.6)$ & \\
\hline Other & $178,555(3.1)$ & $755(2.3)$ & \\
\hline Hypertension & $3,342,609(55.2)$ & $21,505(65.6)$ & $<0.001$ \\
\hline Diabetes Mellitus & $1,623,171(26.8)$ & $10,831(33)$ & $<0.001$ \\
\hline Arrhythmias & $999,060(16.5)$ & $5706(17.4)$ & $<0.001$ \\
\hline Hyperthyroidism & $30,933(0.5)$ & $183(0.6)$ & 0.238 \\
\hline Smoking & $981,827(16.2)$ & $6195(18.9)$ & $<0.001$ \\
\hline Obesity & $955,616(15.8)$ & $8639(26.3)$ & $<0.001$ \\
\hline Alcohol abuse & $1,153,177(19.1)$ & $10,241(31.2)$ & $<0.001$ \\
\hline Cocaine abuse & $84,584(1.4)$ & $347(1.1)$ & $<0.001$ \\
\hline Long term use of corticosteroids & $93,308(1.5)$ & $1283(3.9)$ & $<0.001$ \\
\hline History of chemotherapy/immunotherapy & $85,623(1.4)$ & $480(1.5)$ & 0.461 \\
\hline History of radiotherapy & $81,775(1.4)$ & $493(1.5)$ & 0.02 \\
\hline Sarcoidosis & $16,254(0.3)$ & $158(0.5)$ & $<0.001$ \\
\hline Total & $6,084,184(100)$ & $32,807(100)$ & \\
\hline
\end{tabular}

alcoholic CM (adjusted OR 0.941, 95\% CI 0.651-1.359, $p=0.74)$.

\section{Discussion}

This is a cross sectional study aimed to investigate the association between psoriasis and NIDCM. We found that NIDCM was more prevalent in patients with psoriasis; however, this association is likely due to higher prevalence of risk factors of NIDCM, rather than psoriasis. The only exception was viral $\mathrm{CM}$, which was 2.3 times more likely to affect patients with psoriasis. We also found that arrythmias, followed by hypertension, black race, and male gender were the strongest predictors of NIDCM. Other independent predictors included native American race, alcoholism, sarcoidosis, hyperthyroidism, and cocaine use, among others (Table 2).

Several studies have described the higher prevalence of cardiovascular diseases such as acute coronary disease and cerebrovascular events in psoriatic patients comparing to the general population [5-13]. However, the prevalence of cardiomyopathy in those patients has not been well studied. The prevalence of psoriasis in our study was $0.5 \%$, with mean age of our psoriatic patients was
61 years $( \pm 15.4)$ years comparing to a mean age of 57 $( \pm 20.2)$ years for all cause admission. Regarding ethnicity, Caucasians were more prevalent in our data in both populations. However, psoriasis has been noted to be more prevalent in white race historically [14]. We found an equal distribution between males and females.

Despite several previous studies and data that demonstrated the association of adverse cardiovascular risk factors and events with psoriasis or psoriatic arthritis [5-13], our present study found that no increased occurrence of NIDCM with any type of psoriasis after adjusting for the traditional risk factors such as age, sex, race, diabetes mellitus, hypertension, alcohol abuse, cocaine abuse, arrythmias, and obesity. Therefore, psoriasis cannot be considered as independent risk factor for NIDCM according to our study.

Nonetheless, upon literature review, an interesting relationship between psoriasis and non-ischemic cardiomyopathy has been increasingly reported in few case reports and studies. Most of these cases were associated with non-ischemic dilated cardiomyopathy; however, few reports described hypertrophic cardiomyopathy in psoriasis patients [15-18]. Eliakim-Raz 
Table 2 Multivariable logistic regression model to predict nonischemic cardiomyopathies

\begin{tabular}{llll}
\hline Variable & OR & $95 \%$ Cl for OR & $p$ value \\
\hline Age & 0.989 & $0.988-0.990$ & $<0.001$ \\
Sex, female & 0.472 & $0.462-0.482$ & $<0.001$ \\
Race, White & 1 & & Ref \\
Race, Black & 2.280 & $2.228-2.333$ & $<0.001$ \\
Race, Hispanic & 1.271 & $1.228-1.315$ & $<0.001$ \\
Race, Asian or Pacific Islander & 1.352 & $1.270-1.439$ & $<0.001$ \\
Race, Native American & 1.711 & $1.536-1.906$ & $<0.001$ \\
Race, other & 1.079 & $1.013-1.150$ & 0.018 \\
Diabetes Mellitus & 1.035 & $1.014-1.056$ & 0.001 \\
Hypertension & 2.615 & $2.541-2.690$ & $<0.001$ \\
Alcoholism & 1.639 & $1.593-1.688$ & $<0.001$ \\
Smoking & 1.175 & $1.146-1.205$ & $<0.001$ \\
Cocaine use & 1.429 & $1.347-1.516$ & $<0.001$ \\
Arrhythmias & 5.191 & $5.082-5.302$ & $<0.001$ \\
Obesity & 0.819 & $0.793-0.845$ & $<0.001$ \\
Sarcoidosis & 1.541 & $1.365-1.741$ & $<0.001$ \\
Psoriasis & 1.030 & $0.912-1.165$ & 0.632 \\
History of chemo/immunotherapy & 1.428 & $1.320-1.544$ & $<0.001$ \\
History of radiotherapy & 0.964 & $0.886-1.049$ & 0.391 \\
Hyperthyroidism & 1.498 & $1.362-1.648$ & $<0.001$ \\
\hline
\end{tabular}

Table 3 Bivariate correlations between psoriasis and different types of cardiomyopathies

\begin{tabular}{lccl}
\hline Cardiomyopathy type & Frequency & N with psoriasis (\%) & $p$ value \\
\hline Idiopathic & 39,943 & $239(0.6)$ & 0.11 \\
Peripartum CM & 903 & $1(0)$ & 0.08 \\
Alcoholic CM & 4105 & $36(0.9)$ & 0.003 \\
Drug induced CM & 2626 & $11(0.4)$ & 0.40 \\
Viral CM & 456 & $6(1.3)$ & 0.02 \\
Total NICM & 46,369 & $285(0.6)$ & 0.03 \\
\hline
\end{tabular}

et al. investigated 2292 psoriasis patients in 2008 for cardiomyopathy in one large hospital in Israel. This study reported cardiomyopathy of different types in 20 of these psoriasis patients (a prevalence of $0.87 \%$ ). Ten of the twenty were diagnosed with dilated CM (a prevalence of $0.43 \%$ among psoriasis patients) and most of them had normal coronaries [15]. A case report by Abdelaoui et al. also revealed NIDCM in a patient with a long history of psoriasis [16]. Pietrzak et al. reported dilated cardiomyopathy with a severe ventricular impairment higher than it could result from myocardial ischemia in psoriasis patient [17]. Moreover, Fukuhara et al. reported an association between psoriatic arthritis, Takayasu's disease, and non-ischemic dilated
$\mathrm{CM}$, which supposedly suggests a common underlying immune-mediated inflammatory process among the three conditions [18].

Another cohort study by Zhao et al. demonstrated that patients with severe psoriasis showed subclinical myocardial (left ventricular systolic) dysfunction as detected by $2 \mathrm{D}$ speckle tracking derived strain analysis compared with control group [19]. Milaniuk et al. screened the echocardiography of patients with psoriasis and psoriatic arthritis and reported left ventricle diastolic dysfunction in $27.8 \%$ of the patients, and left ventricle hypertrophy in $11.1 \%$. Left ventricular systolic dysfunction has been noticed as well [20]. Similarly, a large study conducted by Biyik et al. in one of the hospitals in Turkey in 2006 found that the longer is the disease, the higher incidence of left ventricular diastolic dysfunction is in psoriasis patients [21].

Studies have proposed few possible hypotheses that would lead to NIDCM in psoriasis patients [22, 23]. One of them suggests that psoriasis-induced myocardial inflammation will eventually results in a form of inflammatory non-ischemic dilated cardiomyopathy involving certain growth factors and cytokines produced by inflammatory cells such as transforming growth factor- $\beta$, interleukin-1 (IL-1), and interleukin-17A (IL-17A) [22]. Another article by Ryabkova et al. illustrated an interesting autoantibody-mediated mechanism engaging several classes of autoantibodies (AAb) such as cytotoxic autoantibodies or cell-modulating autoantibodies. Cytotoxic antibodies, such as anti-cardiac troponin $\mathrm{I} A \mathrm{~b}$, anti- $\alpha$ - and anti- $\beta$-myosin heavy chain $\mathrm{Ab}$ and anti-mitochondrial Ab, can cause antibody-dependent cardiomyocyte death, accompanied by severe inflammation with increase in the local concentration of tumor necrosis factor (TNF- $\alpha$ ), nitric oxide, and cytokines. On the other hand, cell-modulating autoantibodies have dysregulating effects resulting in alteration of cell function, growth and signaling and eventually leading to contractility and/or conduction disorders [23].

Interestingly, on bivariate analysis of the psoriasis patients in our study, the prevalence of alcoholic and viral cardiomyopathies was higher in this population compared to the general population, at $0.9 \%$ and $1.3 \%$, respectively. Having said that, after adjusting for potential confounding factors in multivariable regression models, only viral CM seemed to maintain the statistical significance (OR 2.3, 95\% CI 1.020-5.131, $p=0.045$ ). The higher prevalence of alcoholic CM in patients with psoriasis can be attributed to the higher prevalence of alcoholism in patients with psoriasis (31\% vs $19 \%$, Table 1). Although not statistically significant, the association between alcoholic CM and psoriasis is noticeably observed at $0.941 \%$ in our study which could be 
attributed to the psychosocial aspects and consequences of this debilitating autoimmune disease [24, 25]. As viral cardiomyopathy is the only type that has been noticed to be associated with psoriasis in our current study, several explanations could justify this higher incidence. One of the reasons is that psoriasis patients have immune dysfunction that could put them at a higher risk for infections and its complications, including viral infections, compared to the general population [26].

Our study is one of the first studies to highlight the association between NIDCM and any type of psoriasis; therefore, we cannot disclose a definitive conclusion, especially given the cross-sectional design of the study that is insufficient to establish causality. More robust and larger studies could illustrate the causality between viral and alcoholic cardiomyopathies and psoriasis. In addition, including inflammatory markers in future studies can provide additional information about the existence of such association.

We observe that our study has several limitations that might impact the application of its findings and must be acknowledged. First, severity of the disease in psoriatic patients was not available, therefore the association between severity of psoriasis and cardiomyopathy cannot be assessed. Second, HCUP-NIS database does not provide information on other important confounders such as anti-psoriatic immunosuppressive medications that could cause cardiomyopathy as a side effect. Finally, our study is cross sectional which analyzes the prevalence at single point time and subsequently cannot assess the influence of the disease duration on precipitating cardiomyopathy.

\section{Conclusion}

Psoriasis is a chronic autoimmune disorder which traditionally carries a high cardiovascular risk including accelerated atherosclerosis and ischemic CM in comparison to the general population. Moreover, previous data has suggested that aggressive treatment of the disease as well as better control of the traditional atherosclerotic risk factors may reduce cardiovascular events in psoriatic patients. Despite the interesting relation between psoriasis and NIDCM that has been reported increasingly in few case reports and studies, our current study indicated no correlation between psoriasis and NIDMC, except viral type, although we cannot reach a definitive conclusion from a single study and the aforementioned limitations might have influenced the results. Therefore, further research especially prospective studies are recommended to illuminate the exact correlation between all different subclasses of psoriasis and non-ischemic dilated cardiomyopathy.

\section{Appendix}

List of ICD-10 used in the study:

1. Psoriasis: "L40" and its subclassifications.

2. Peripartum cardiomyopathy: "O90.3".

3. Viral cardiomyopathy: "B33.24".

4. Idiopathic dilated cardiomyopathy: "I42.0"

5. Alcoholic cardiomyopathy: "I42.6"

6. Drug induced cardiomyopathy: "I42.7"

7. Non-ischemic cardiomyopathies: ICD-10 of points 2-6 above.

8. Diabetes mellitus: "E08", “E09", “E10”, “E11", “E12", "E13" and their subclassifications.

9. Hypertension: "I10", “I11", “I12", "I13", “I15" and their subclassifications.

10. Arrhythmias: "I47", "I48" and their subclassifications.

11. Alcohol: "F10" and its subclassification.

12. Cocaine: "F14" and its subclassification.

13. Obesity: "E66", "Z68.3", "Z68.4" and subclassifications.

14. Smoking: "F17" and subclassifications.

15. Sarcoidosis: "D86" and subclassifications.

16. Hyperthyroidism: "E05" and subclassifications.

17. Chemo/Immunotherapy: "Z92.25", "Z92.21".

18. Radiotherapy: "Z92.3".

Abbreviations

NIDCM: Non-ischemic dilated cardiomyopathy; IRB: Institutional review board; CM: Cardiomyopathy.

Acknowledgements

Not applicable.

Authors' contributions

AA, NA, SD, and SP: Conceptualization; AA: methodology; AA, NA, SD: analysis; AA, NA, SD: data curation; AA, NA, SD: writing —original draft preparation; AA, $N A, S D, S P, M H, D R A$, and DC: writing - review and editing; SP, MH, DRA, and DC: supervision. All authors have read and agreed to the published version of the manuscript. All authors read and approved the final manuscript.

\section{Funding}

This research did not receive any specific grant from funding agencies in the public, commercial, or not-for-profit sectors.

\section{Availability of data and materials}

The datasets generated and/or analyzed during the current study are available in the Healthcare Cost and Utilization Project National Inpatient Sample (HCUP-NIS) 2017 database repository (https://www.hcup-us.ahrg.gov/nisov erview.jsp).

\section{Declarations}

Ethics approval and consent to participate

Institutional review board (IRB) is not required to for studies utilizing the national inpatient sample, as it falls under the "limited data sets" category exempted by the HIPAA privacy regulations (http://privacyruleandresearch. 
nih.gov). The study was conducted in agreement with the principles of the Declaration of Helsinki.

\section{Consent for publication}

Not applicable.

\section{Competing interests}

The authors declare that they have no competing interests.

\section{Author details}

${ }^{1}$ Department of Medicine, Jersey Shore University Medical Center, 1945 Route 33, Neptune, NJ 07753, USA. ${ }^{2}$ Department of Rheumatology, Jersey Shore University Medical Center, Neptune, NJ, USA. ${ }^{3}$ Department of Cardiology, Jersey Shore University Medical Center, Neptune, NJ, USA.

Received: 6 February 2021 Accepted: 30 March 2021

Published online: 31 March 2021

\section{References}

1. Michalek IM, Loring B, John SM. A systematic review of worldwide epidemiology of psoriasis. J Eur Acad Dermatol Venereol. 2017;31(2):205-12. https://doi.org/10.1111/jdv.13854

2. Benhadou F, Mintoff D, Schnebert B, Thio HB. Psoriasis and microbiota: a systematic review. Diseases. 2018;6(2):47. https://doi.org/10.3390/disea ses6020047.

3. Taylor SL, Petrie M, O'Rourke KS, Feldman SR. Rheumatologists'recommendations on what to do in the dermatology office to evaluate and manage psoriasis patients'joint symptoms. J Dermatol Treat. 2009;20(6):350-3. https://doi.org/10.3109/09546630902817887.

4. Pearce DJ, Morrison AE, Higgins KB, Crane MM, Balkrishnan R, Fleischer AB Jr, Feldman SR. The comorbid state of psoriasis patients in a university dermatology practice. J Dermatol Treat. 2005;16(5-6):319-23. https://doi. org/10.1080/09546630500335977.

5. Kimhi O, Caspi D, Bornstein N, Maharshak N, Gur A, et al. Prevalence and risk factors of atherosclerosis in patients with psoriatic arthritis. Semin Arthritis Rheum. 2007;36(4):203-9. https://doi.org/10.1016/j.semarthrit. 2006.09.001.

6. Kimball AB, Robinson D Jr, Wu Y, Guzzo C, Yeilding N, Paramore C, Fraeman K, Bala M. Cardiovascular disease and risk factors among psoriasis patients in two US healthcare databases, 2001-2002. Dermatology. 2008;217:27-37. https://doi.org/10.1159/000121333.

7. Gladman DD, Ang M, Su L, et al. Cardiovascular morbidity in psoriatic arthritis. Ann Rheum Dis. 2009;68:1131-5. https://doi.org/10.1136/ard. 2008.094839

8. Gulati AM, Semb AG, Rollefstad S, et al. On the HUNT for cardiovascular risk factors and disease in patients with psoriatic arthritis: populationbased data from the Nord-Trøndelag health study. Ann Rheum Dis. 2016;75(5):819-24. https://doi.org/10.1136/annrheumdis-2014-206824.

9. Khraishi M, Aslanov R, Rampakakis E, Pollock C, Sampalis JS. Prevalence of cardiovascular risk factors in patients with psoriatic arthritis. Clin Rheumatol. 2014;33(10):1495-500. https://doi.org/10.1007/s10067-014-2743-7.

10. Ogdie A, Yu Y, Haynes K, et al. Risk of major cardiovascular events in patients with psoriatic arthritis, psoriasis and rheumatoid arthritis: a population-based cohort study. Ann Rheum Dis. 2015;74:326-32. https:// doi.org/10.1136/annrheumdis-2014-205675.
11. Kristensen SL, McInnes IB, Sattar N. Psoriasis, psoriatic arthritis and cardiovascular risk: are we closer to a clinical recommendation? Ann Rheum Dis. 2015;74:321-2. https://doi.org/10.1136/annrheumdis-2014-206617.

12. Yim KM, Armstrong AW. Updates on cardiovascular comorbidities associated with psoriatic diseases: epidemiology and mechanisms. Rheumatol Int. 2017;37(1):97-105. https://doi.org/10.1007/s00296-016-3487-2.

13. Kibari A, Cohen AD, Gazitt T, et al. Cardiac and cardiovascular morbidities in patients with psoriatic arthritis: a population-based case control study. Clin Rheumatol. 2019;38:2069-75. https://doi.org/10.1007/ s10067-019-04528-y.

14. Baeta I, Bittencourt F, Gontijo B, et al. Comorbidities and cardiovascular risk factors in patients with psoriasis. An Bras Dermatol. 2014;89(5):73544. https://doi.org/10.1590/abd1806-4841.20142874.

15. Eliakim-Raz N, Shuvy M, Lotan C, Planer D. Psoriasis and dilated cardiomyopathy: coincidence or associated diseases? Cardiology. 2008;111(3):2026. https://doi.org/10.1159/000121605.

16. Abdelaoui B, Benlafkih O, Ztati M, Karimi S, et al. The diagnosis of dilated cardiomyopathy during extended psoriasis: case report. Int J Adv Res. 2020;8(01):596-600. https://doi.org/10.21474/IJAR01/10335.

17. Pietrzak A, Brzozowska A, Lotti T, Mosiewicz J, Wysokiński A, et al. Psoriasis and cardiovascular. Dermatol Ther. 2013;26:489-92. https://doi.org/10. 1111/dth.12021.

18. Fukuhara K, Urano Y, Akaike M, Ahsan K, Arase S. Psoriatic arthritis associated with dilated cardiomyopathy and Takayasu's arteritis. Br J Dermatol. 1998;138:329-33. https://doi.org/10.1046/j.1365-2133.1998.02085.x.

19. Zhao C-T, Yeung C-K, Siu C-W, Tam S, Chan J, Chen Y, Chan H-H, Tse H-F, Yiu K-H. Relationship between parathyroid hormone and subclinical myocardial dysfunction in patients with severe psoriasis. J Eur Acad Dermatol Venereol. 2014;28:461-8. https://doi.org/10.1111/jdv.12123.

20. Milaniuk S, Pietrzak A, Mosiewicz B, Mosiewicz J, Reich K. Influence of psoriasis on circulatory system function assessed in echocardiography. Arch Dermtol Res. 2015;307(10):855-61. https://doi.org/10.1007/ s00403-015-1586-7.

21. Biyik I, Narin A, Bozok M, Ergene O. Echocardiographic and clinical abnormalities in patients with psoriasis. J Int Med Res. 2006;34(6):632-9. https:// doi.org/10.1177/147323000603400608.

22. Hashim T, Ahmad A, Chaudry A, Khouzam R. Psoriasis and cardiomyopathy: a review of the literature. South Med J. 2017;110(2):97-100. https:// doi.org/10.14423/SMJ.0000000000000603.

23. Ryabkova VA, Shubik YV, Erman MV, Churilov LP, Kanduc D, Shoenfeld Y. Lethal immunoglobulins: autoantibodies and sudden cardiac death. Autoimmun Rev. 2019;18(4):415-25. https://doi.org/10.1016/j.autrev. 2018.12.005.

24. Prussick L, Jimenez E, Nussbaum D, Prussick R. Psoriasis and psychological comorbidities. J Psoriasis Psoriatic Arthritis. 2016;1:80-5. https://doi.org/ 10.1177/247553031600100206

25. Svanström C, Lonne-Rahm SB, Nordlind K. Psoriasis and alcohol. Psoriasis. 2019;9:75-9. https://doi.org/10.2147/PTT.S164104.

26. Rademaker M, Agnew K, Anagnostou N, et al. Psoriasis and infection. A clinical practice narrative. Australas J Dermatol. 2019;60(2):91-8. https:// doi.org/10.1111/ajd.12895.

\section{Publisher's Note}

Springer Nature remains neutral with regard to jurisdictional claims in published maps and institutional affiliations. 